\section{Depressed-type early duodenal carcinoma (carcinoma in situ) observed by enhanced magnification endoscopy}

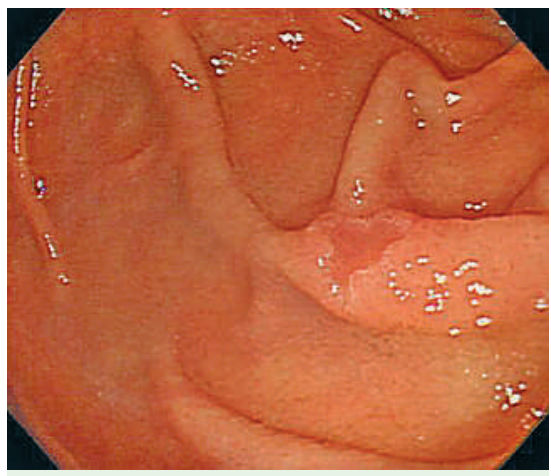

Figure 1 Endoscopy showed a depressedtype tumor in the descending duodenum.

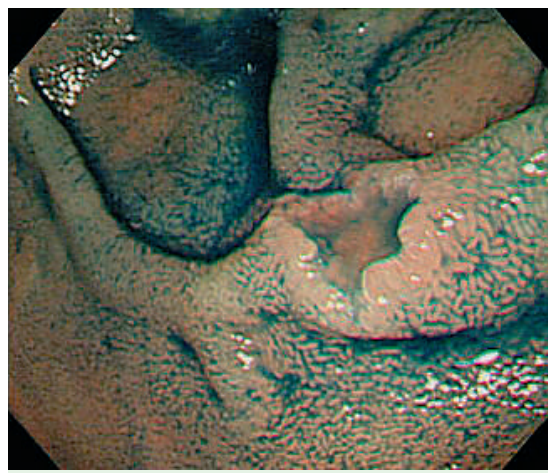

Figure 2 Chromoendoscopy with $0.2 \%$ indigo carmine more clearly revealed demarcation of this lesion and surrounding villi.

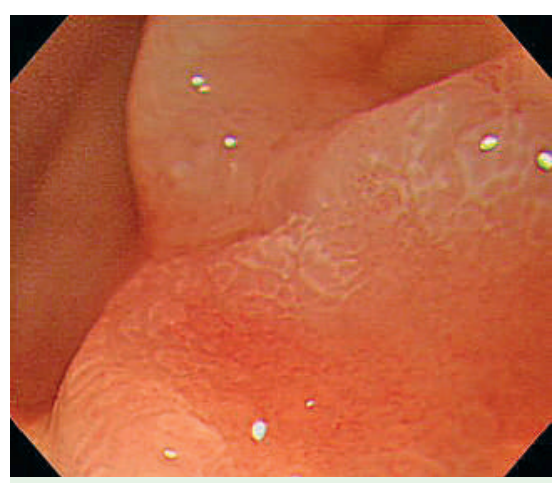

Figure 3 Conventional magnification endoscopy revealed irregular microvessels in the smooth depression.

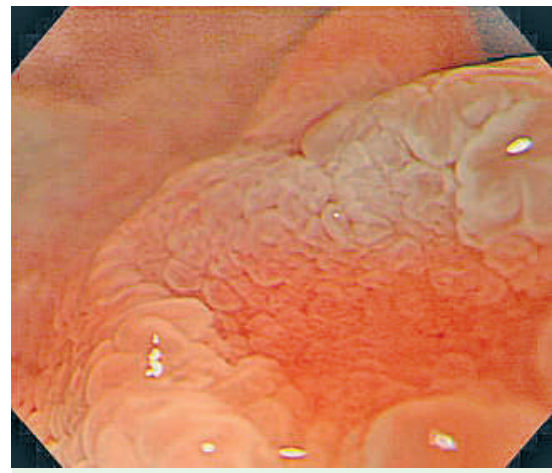

Figure 4 Enhanced magnification endoscopy (with $1.5 \%$ acetic acid) revealed an irregular fine microstructure in the depressed lesion.

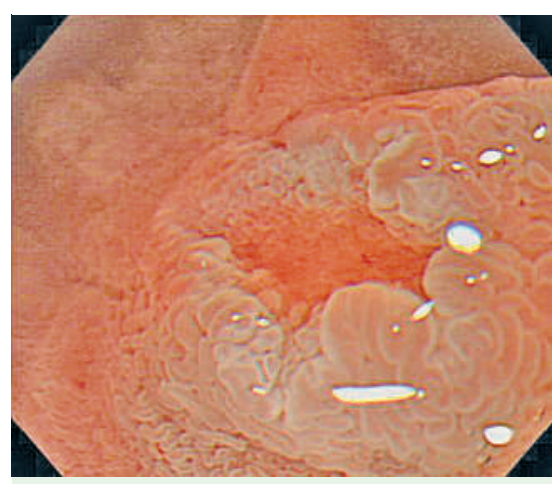

Figure 5 Enhanced magnification endoscopy (with $1.5 \%$ acetic acid) showed normal villi surrounding the depressed lesion.
Early primary nonampullary duodenal carcinoma is an extremely rare disease. We observed the features of depressedtype early duodenal carcinoma by enhanced magnification endoscopy (EME). A 67-year-old woman underwent gastrointestinal endoscopy because of epigastralgia. Endoscopy showed a depressed lesion, 3-5 $\mathrm{mm}$ in diameter, in the descending duodenum on the opposite side of the ampulla of Vater ( Figure 1 ). Chromoendoscopy with $0.2 \%$ indigo carmine revealed a clear demarcation of this lesion and surrounding villi ( $\bullet$ Figure 2 ). Conventional magnification endoscopy revealed microvessels in the depression (๑ Figure 3 ). EME with $1.5 \%$ acetic acid clearly revealed an irregular microstructure in the depressed lesion and surrounding normal villi ( $\bullet$ Figure 4 and - Figure 5). Histological analysis of the biopsy specimen revealed an adenocarcinoma of duodenum. We performed an endoscopic mucosectomy, and the lesion was completely resected without complication.

A cross section of the tumor specimen was identified as a depressed type $(\bullet$ Figure 6), and the margins were carcinoma free. Histopathologically, the lesion was diagnosed as a well-differentiated adenocarcinoma limited to the mucosa ( $\bullet$ Figure 6).

Wakabayashi et al. reported that magnifying endoscopy with methylene blue staining seemed to be useful in the diagnosis of duodenal cancer [1]. FriedrichRust et al. reported an early duodenal carcinoma identified using magnification

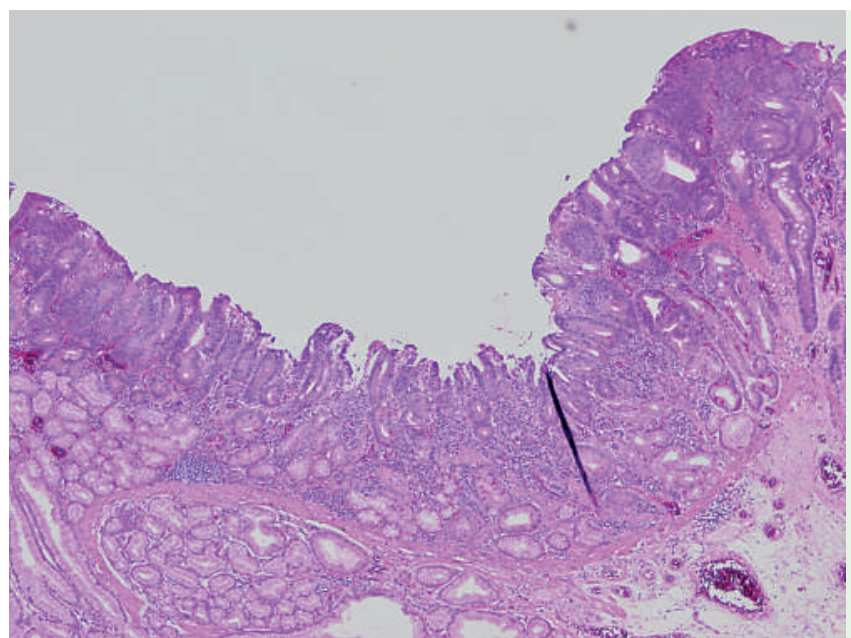

Figure 6 Microscopic image of mucosectomy specimen shows depressed-type well-differentiated adenocarcinoma limited to the mucosa $(H \& E, \times 40)$. 
endoscopy to demarcate and detect neoplastic change in the architecture of the intestinal villi [2].

EME is a useful method for observing mucosal surface microstructures in Barrett's esophagus [3,4] and stomach [5]. However, there have been no reports on the features of the duodenal carcinoma using EME. In the case report discussed here, EME was useful for observing the fine surface structure of the lesion. Indeed we found that there was a correlation between the irregular microstructure and the pathological features of the lesion. EME may be useful for defining the clinicopathologic features of early duodenal carcinoma.

Endoscopy_UCTN_Code_CCL_1AB_2AZ_3AB

\section{K. Tanaka ${ }^{1}$, H. Toyoda ${ }^{1}$, H. Inoue ${ }^{2}$,} Y. Hamada ${ }^{2}$, M. Aoki' ${ }^{2}$, R. Kosaka ${ }^{2}$, M. Takamura ${ }^{3}$, I. Imoto ${ }^{1}$

1 Department of Endoscopic Medicine, Mie University School of Medicine, Tsu, Japan

2 Department of Gastroenterology, Mie University School of Medicine, Tsu, Japan

${ }^{3}$ Department of Pathology, Mie University School of Medicine, Tsu, Japan

\section{References}

1 Wakabayashi H, Murayama H, Kojima Tet al. A case of early duodenal cancer; new endoscopic findings through magnifying endoscopy combined with methylene blue staining method [in Japanese with English abstract]. Gastroenterol Endosc 1984; 26 : 447-462

2 Friedrich-Rust M, Jaeger C, Gossner L et al. Early duodenal adenocarcinoma arising in gastric metaplasia treated by endoscopic resection. Z Gastroenterol 2006; 44: 323 328

3 Guelrud M, Herrera I, Essenfeld $H$ et al. Enhanced magnification endoscopy: a new technique to identify specialized intestinal metaplasia in Barrett's esophagus. Gastrointest Endosc 2001; 53: 559-565

4 Toyoda H, Rubio C, Befrits $R$ et al. Detection of intestinal metaplasia in distal esophagus and esophagogastric junction by enhancedmagnification endoscopy. Gastrointest Endosc 2004; 59: 15-21

5 Tanaka K, Toyoda H, Kadowaki S et al. Features of early gastric cancer and gastric adenoma by enhanced-magnification endoscopy. J Gastroenterol 2006; 41: 332 - 338
Bibliography

DOI $10.1055 / \mathrm{s}-2007-966171$

Endoscopy 2007; 39: E125-E126

(c) Georg Thieme Verlag KG Stuttgart · New York . ISSN 0013-726X

Corresponding author

\section{K. Tanaka, MD}

Department of Endoscopic Medicine Mie University School of Medicine 2-174 Edobashi

Tsu

Mie 514-8507

Japan

Fax: +81-59-231-5200

kyosuket@qa2.so-net.ne.jp 